\title{
Quantification of the influence of external vibrations on the measurement error of a Coriolis mass-flow meter
}

\author{
L. van de Ridder ${ }^{\text {a,* }}$, W.B.J. Hakvoort ${ }^{\mathrm{a}, \mathrm{b}}$, J. van Dijk ${ }^{\mathrm{a}}$, J.C. Lötters ${ }^{\mathrm{a}, \mathrm{c}}$, A. de Boer ${ }^{\mathrm{a}}$ \\ ${ }^{a}$ University of Twente, Faculty of Engineering Technology, P.O. Box 217, 7500AE Enschede, The Netherlands \\ ${ }^{\mathrm{b}}$ DEMCON Advanced Mechatronics, Enschede, The Netherlands \\ c Bronkhorst High-Tech B.V., Ruurlo, The Netherlands
}

\section{A R T I C L E I N F O}

Article history:

Received 18 March 2014

Received in revised form 9 July 2014

Accepted 6 August 2014

Available online 29 August 2014

Keywords:

Coriolis mass-flow meter

External vibrations

Power Spectral Density

Transfer function

\begin{abstract}
A B S T R A C T
In this paper the influence of external vibrations on the measurement value of a Coriolis mass-flow meter (CMFM) for low flows is investigated and quantified. Model results are compared with experimental results to improve the knowledge on how external vibrations affect the mass-flow measurement value. A flexible multi-body model is built and the working principle of a CMFM is explained. Some special properties of the model are evaluated to get insight into the dynamic behaviour of the CMFM. Using the model, the transfer functions between external vibrations (e.g. floor vibrations) and the flow error are derived. The external vibrations are characterised with a PSD. Integrating the squared transfer function times the PSD over the whole frequency range results in an RMS flow error estimate. In an experiment predefined vibrations are applied on the casing of the CMFM and the error is determined. The experimental results show that the transfer functions and the estimated measurement error correspond with the model results.

The agreement between model and measurements implies that the influence of external vibrations on the measurement is fully understood. This result can be applied in two ways; firstly that the influence of any external vibration spectrum on the flow error can be estimated and secondly that the performance of different CMFM designs can be compared and optimised by shaping their respective transfer functions.
\end{abstract}

(c) 2014 Elsevier Ltd. All rights reserved.

\section{Introduction}

A Coriolis mass-flow meter (CMFM) is an active device based on the Coriolis force principle for direct mass-flow measurements with a high accuracy, range-ability and repeatability [1]. The working principle of a CMFM is as follows: a fluid conveying tube is actuated to oscillate with a low amplitude at a resonance frequency in order to minimise the amount of supplied energy. A fluid flow in the vibrating tube induces Coriolis forces, proportional to the mass-flow, which affect the tube motion and change the mode shape. Measuring the tube displacement, such that the change of its mode shape is determined, allows calculating the mass-flow.

Besides the sensitivity for a mass-flow, there are many factors influencing the measurement value. Anklin et al. [1] mentioned several factors: the effect of temperature and flow profiles on the sensitivity and measurement value, external vibrations and flow pulsations. More factors are investigated by Enz et al. [2]: Flow

\footnotetext{
* Corresponding author.

E-mail address: L.vanderidder@utwente.nl (L. van de Ridder).
}

pulsations, asymmetrical actuator and detector positions and structural non-uniformities. And more recent also by Kazahaya [3]: uneven flow rates in two flow tubes, vibration effects, temperature effects and the inner pressure effects. Further Bobovnik et al. [4] studied the effect of disturbed velocity profiles due to installation effects and other influencing factors like two-phase or even three-phase flow effects were studied by Henry et al. [5].

In our research we focus mainly on the effect of floor/mechanical/external vibrations. These vibrations create additional components in the CMFM sensor signals [6], those additional components can introduce a measurement error. The effect of mechanical vibrations on the sensor response of a CMFM is also studied by Cheesewright $[7,8]$. The analytical study showed that external vibrations at the meter's drive frequency produces a measurement error, regardless of the flow measurement algorithm. There is no attempt made to quantify the error in any particular meter, since such an error depends on dimensions, type of actuators and sensors and the used flow measurement algorithm.

A solution to reduce the influence of external vibrations is to apply a robust balancing system. (e.g. a twin tube configuration) $[1,3]$. There are many types of CMFMs available, whereby the size depends on the flow range. One category is the CMFM for low 
flows [9]. For low flows, the Coriolis force induced motion is relatively small compared to external vibrations induced motions, thus CMFM's designed to be sensitive to low flows is rather sensitive to external vibrations. Applying a twin tube configuration is not an option, because some structural non-uniformities [2] can lead to large differences between the two tubes, due to their small dimensions. This has a negative impact on the measurement sensitivity of the instrument and reduces the decoupling of external vibrations to the internal measurement system.

A quantitative model of the influence of external vibrations is not yet available. In this study the effect of external vibrations on the measurement error is quantified using an experimentally validated model. The results presented in this study are an extension of previous work [10]. First, a model of a CMFM is derived, using the multi-body package SPACAR [11] resulting in a linear state space representation [12]. In the modelling, a tubeelement [13] is used to model the inertial interaction between flow and the tube dynamics. Secondly, the model is extended to be able to predict the influence of external vibrations, with the eventual goal to find and test designs that reduce the influence of external vibrations on an erroneous mass-flow reading.

\section{Modelling method}

In this section, the Finite Element Method (FEM) model is explained. Subsequently, the system equations are derived and the inputs and outputs are defined to derive the input-output relations. This results in a state space representation of a CMFM in the final subsection.

\subsection{Coriolis mass-flow meter}

For this research a functional model of the patented design $[9,14]$ (see Fig. 1) is used. First, a FEM model is derived, using the multi-body package SPACAR [11]. The graphical representation of the model is shown in Fig. 2. The model consists of a tube-window, conveying the fluid flow, which is actuated by two actuators act and $a_{c t}$. The displacements of the flexible tube-window are measured by two displacements sensors $s_{1}$ and $s_{2}$. On the casing a vector $\boldsymbol{a}_{0}$, representing the external vibrations and consisting of three translation and three rotational movements, is imposed. The model is made out of multi-body beam, truss and tube elements. The beam elements are used to model the rigid casing and the truss elements to measure relative displacements and to apply a force on the tube-window. Further, a tube-element [13] is used to model the inertial interaction between flow and the tube dynamics.

\subsection{System equations}

The linearised system equations of the FEM model, with $n$ degrees of freedom of tube deformations $\boldsymbol{q}$ and the imposed casing movements (rheonomic degrees of freedom: $\boldsymbol{x}_{0}, \boldsymbol{v}_{0}=\dot{\boldsymbol{x}}_{0}, \boldsymbol{a}_{0}=\ddot{\boldsymbol{x}}_{0}$ ), can be written as [12]:

$\left[\begin{array}{ll}\boldsymbol{M}_{11} & \boldsymbol{M}_{12} \\ \boldsymbol{M}_{21} & \boldsymbol{M}_{22}\end{array}\right]\left[\begin{array}{c}\ddot{\boldsymbol{q}} \\ \boldsymbol{a}_{0}\end{array}\right]+[\boldsymbol{C}(\dot{\Phi})+\boldsymbol{D}]\left[\begin{array}{c}\dot{\boldsymbol{q}} \\ \boldsymbol{v}_{0}\end{array}\right]+\left[\boldsymbol{K}+\boldsymbol{N}\left(\dot{\Phi}^{2}\right)\right]\left[\begin{array}{c}\boldsymbol{q} \\ \boldsymbol{x}_{0}\end{array}\right]=\left[\begin{array}{c}\boldsymbol{f} \\ \boldsymbol{F}_{0}\end{array}\right]$

The other terms are the mass matrix $\boldsymbol{M}$, stiffness matrix $\boldsymbol{K}$, damping matrix $\boldsymbol{D}$, the velocity sensitive matrix $\boldsymbol{C}$, the dynamic stiffness matrix $\boldsymbol{N}$, the actuation input vector $\boldsymbol{f}$ and the reaction force $\boldsymbol{F}_{0}$. The matrices $\boldsymbol{C}$ and $\boldsymbol{N}$ depend linear and quadratic on the mass-flow $\dot{\Phi}$ respectively, and are representing the forces induced

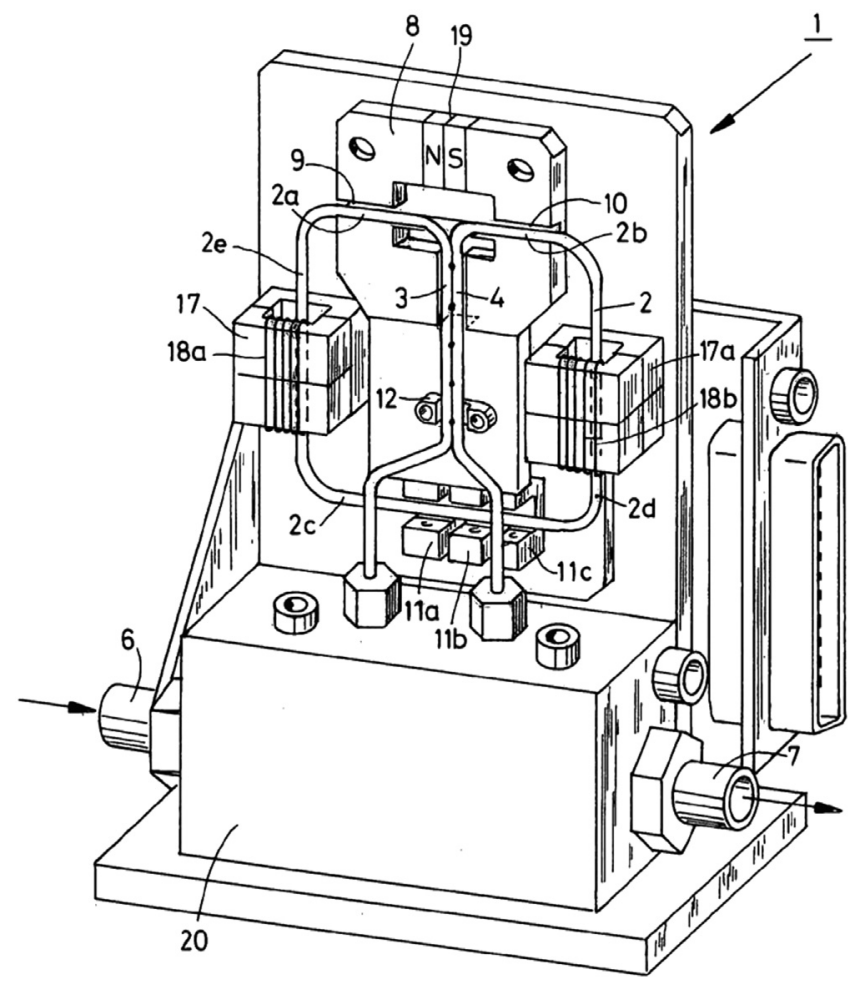

Fig. 1. Coriolis mass-flow meter, used as a reference instrument in this study Details on the patented design are given in $[9,14]$. The instrument is connected to a pipeline; a fluid flow enters the instrument (6), flows trough the tube-window (2) and exits the instrument (7). The flexible tube-window (2) is actuated in resonance by an Lorentz actuator (8) and the displacements are measured by optical displacements sensors (11abc) [15].

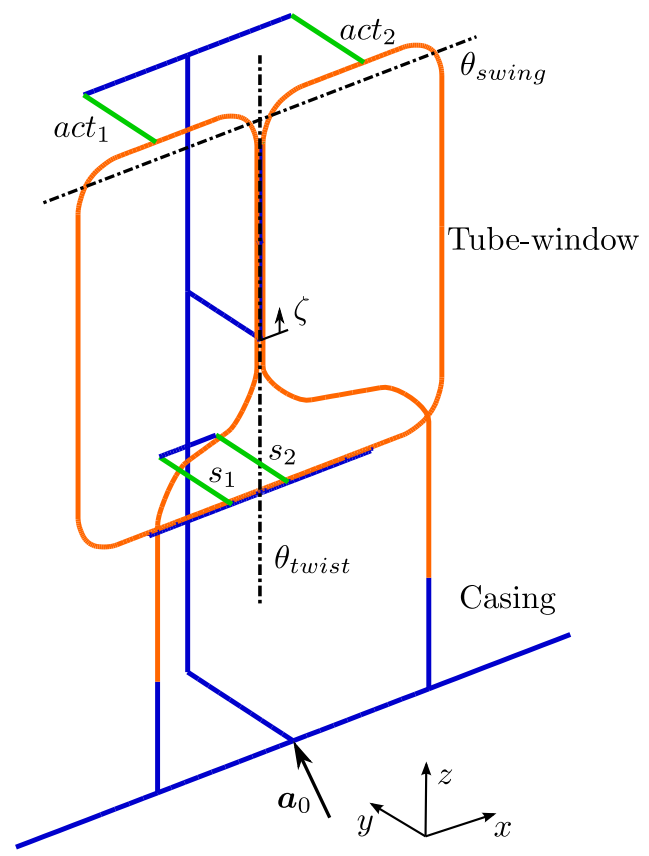

Fig. 2. CMFM multi-body model, the flexible tube-window is actuated by two Lorentz actuators $a c t_{1}$ and $a c t_{2}$. The trajectory of the curved tube-window is parametrised by $\zeta$, starting at the fixation point of the tube-window to the casing. The displacement are measured by two displacements sensors $s_{1}$ and $s_{2}$. On the casing a vector $\boldsymbol{a}_{0}$ with floor movements is imposed.

by respectively the Coriolis and centrifugal acceleration of the flow. The matrices $\boldsymbol{C}, \boldsymbol{D}, \boldsymbol{K}$ and $\boldsymbol{N}$ can be divided into the same parts as the mass matrix $\boldsymbol{M}$. Using the multi-body package SPACAR 
[11] the system matrices with respect to the element deformations and the imposed floor movements of the model are derived.

The matrices $\boldsymbol{D}_{12}, \boldsymbol{K}_{12}$ and their transposed matrices appear to be zero, due to the choice of element deformations as degrees of freedom. (E.g. $\boldsymbol{K}_{12}=\mathbf{0}$, because there is no coupling between the location of the casing $\boldsymbol{x}_{0}$ and the internal deformations $\mathbf{q}$.)

The casing motion is prescribed and thus the only dynamic degrees of freedom are the tube deformations, for which the equations of motion are derived from the top row of Eq. (1):

$\boldsymbol{M}_{11} \ddot{\boldsymbol{q}}=\boldsymbol{f}+\boldsymbol{f}_{\text {dis }}-\boldsymbol{C}_{11} \dot{\boldsymbol{q}}-\boldsymbol{D}_{11} \dot{\boldsymbol{q}}-\boldsymbol{K}_{11} \boldsymbol{q}-\boldsymbol{N}_{11} \boldsymbol{q}$

including a external disturbance force, consisting of imposed external accelerations:

$\boldsymbol{f}_{\text {dis }}=-\boldsymbol{M}_{12} \boldsymbol{a}_{0}$

whereby $\boldsymbol{C}_{12} \boldsymbol{v}_{0}$ and $\boldsymbol{N}_{12} \boldsymbol{x}_{0}$ are omitted, because their magnitude is orders lower then $\boldsymbol{M}_{12} \boldsymbol{a}_{0}$. The vector of imposed external accelerations, three translations and three rotations, is equal to: $\boldsymbol{a}_{0}=\left\{\begin{array}{llllll}a_{x} & a_{y} & a_{z} & \alpha_{R x} & \alpha_{R z} & \alpha_{R z}\end{array}\right\}^{T}$

To gain more insight into the model, the degrees of freedom are reduced by applying a modal reduction method. For the modal reduction, the eigenvalue problem $\left(\boldsymbol{K}_{11}+\boldsymbol{N}_{11}-\omega_{i}^{2} \boldsymbol{M}_{11}\right) \boldsymbol{v}_{i}=0$ is solved, which results in natural frequencies $\omega_{i}$ and the corresponding eigenvector $\boldsymbol{v}_{i}$, the mode shape. The equations of motion are rewritten in the modal coordinates, defined as: $\boldsymbol{q}=\boldsymbol{V} \boldsymbol{z}$, where $\boldsymbol{V}=\left[\boldsymbol{v}_{1}, \boldsymbol{v}_{2}, \ldots, \boldsymbol{v}_{n}\right]$ is a matrix, normalised such that $\boldsymbol{V}^{T} \boldsymbol{M}_{11} \boldsymbol{V}=\boldsymbol{I}$, of the first $n$ mode shapes and $\boldsymbol{z}$ is the vector of modal amplitudes. Eq. (2) can now be written as

$\ddot{\boldsymbol{z}}+\boldsymbol{V}^{T} \boldsymbol{C}_{11}(\dot{\Phi}) \mathbf{V} \dot{\boldsymbol{z}}+\boldsymbol{V}^{T} \boldsymbol{D}_{11} \mathbf{V} \dot{\boldsymbol{z}}+\boldsymbol{V}^{T} \boldsymbol{K}_{11} \boldsymbol{V} \boldsymbol{z}+\boldsymbol{V}^{T} \boldsymbol{N}_{11}\left(\dot{\Phi}^{2}\right) \boldsymbol{V} \boldsymbol{z}=\boldsymbol{V}^{T} \boldsymbol{f}+\boldsymbol{V}^{T} \boldsymbol{f}_{d i s}$

The reaction forces on the floor can be derived from the lower row of Eq. (1):

$\boldsymbol{F}_{0}=\boldsymbol{M}_{21} \ddot{\boldsymbol{q}}+\boldsymbol{C}_{21} \dot{\boldsymbol{q}}+\boldsymbol{N}_{21} \boldsymbol{q}+\boldsymbol{M}_{22} \boldsymbol{a}_{0}+\left(\boldsymbol{C}_{22}+\boldsymbol{D}_{22}\right) \boldsymbol{v}_{0}+\left(\boldsymbol{K}_{22}+\boldsymbol{N}_{22}\right) \boldsymbol{x}_{0}$

\subsubsection{Actuation}

The flexible tube-window is actuated to have an oscillation around the $\theta_{\text {twist }}$-axis (see Fig. 2), therefore in the model a moment is applied by two forces between the tube and the casing. In model terms the actuator input is equal to

$\boldsymbol{f}=\frac{1}{r_{M}}\left(\boldsymbol{\Gamma}_{a c t_{1}}-\boldsymbol{\Gamma}_{a c t_{2}}\right) M_{a c t}$

where $\boldsymbol{\Gamma}_{a c t_{1}}$ and $\boldsymbol{\Gamma}_{a c t_{2}}$ are vectors with the elongation of the actuator element with respect to the coordinates $\boldsymbol{q}$ of the model, $r_{M}$ the distance between the two actuator elements and $M_{a c t}$ the actuator moment input.

\subsubsection{Sensing}

The movement of the tube-window is measured by two sensors, $s_{1}$ and $s_{2}$. In model terms the sensor displacements are equal to

$s_{i}=\boldsymbol{\Gamma}_{s_{i}} \boldsymbol{q}=\boldsymbol{\Gamma}_{s_{i}} \boldsymbol{V z}$

where $\boldsymbol{\Gamma}_{s_{i}}$ is a vector with the elongation of the $i$ th sensor element with respect to the coordinates $\boldsymbol{q}$ of the model.

\subsection{State space description}

Combining the equations of the previous sections, a state space representation of the CMFM with a state vector $\boldsymbol{x}=[\boldsymbol{z} \dot{\boldsymbol{z}}]^{T}$, input vector $\boldsymbol{u}=\left[\begin{array}{ll}M_{a c t} & \boldsymbol{a}_{0}\end{array}\right]^{T}$ and output vector $\boldsymbol{y}=\left[\begin{array}{ll}s_{1} & s_{2}\end{array}\right]^{T}$ is derived:

$$
\begin{aligned}
\dot{\boldsymbol{x}}= & {\left[\begin{array}{cc}
\mathbf{0} & \boldsymbol{I} \\
-\boldsymbol{V}^{T}\left(\boldsymbol{K}_{11}+\boldsymbol{N}_{11}\left(\dot{\Phi}^{2}\right)\right) \boldsymbol{V} & -\boldsymbol{V}^{T}\left(\boldsymbol{C}_{11}(\dot{\boldsymbol{\Phi}})+\boldsymbol{D}_{11}\right) \boldsymbol{V}
\end{array}\right] \boldsymbol{x} } \\
& +\left[\begin{array}{cc}
\mathbf{0} & \mathbf{0} \\
\boldsymbol{V}^{T} \frac{1}{r_{M}}\left(\boldsymbol{\Gamma}_{a c t_{1}}-\boldsymbol{\Gamma}_{a c t_{2}}\right) & -\boldsymbol{V}^{T} \boldsymbol{M}_{12}
\end{array}\right] \boldsymbol{u} \\
\boldsymbol{y}= & {\left[\begin{array}{ll}
\boldsymbol{\Gamma}_{s_{1}} \boldsymbol{V} & \mathbf{0} \\
\boldsymbol{\Gamma}_{s_{2}} \boldsymbol{V} & \mathbf{0}
\end{array}\right] \boldsymbol{x}+[\mathbf{0}] \boldsymbol{u} }
\end{aligned}
$$

This state space model can be used to investigate the tubewindow displacements as a result of an actuation moment, mass-flow and external vibrations.

\section{Model evaluation}

In this section the model, derived by the method described in Section 2, is evaluated. First, it is shown that the modal decomposition gives a good understanding of the dynamic behaviour of a CMFM. A distinction is made between model results with and without a mass-flow. Second, the mass-flow measurement value is related to the mode shapes. Also, it is explained how the massflow is determined in practice by phase demodulation. In the third subsection, the effect of external vibrations on the flow measurement is shown, yielding a transfer function from external vibrations to the mass-flow measurement. The final subsection shows how the influence of broadband external vibrations on the RMS mass-flow measurement value can be calculated.

\subsection{Modal decomposition}

In Section 2 a dynamic model of a CMFM is derived. From the model, mass and stiffness matrices are obtained. Solving the eigenvalue problem $\left(\boldsymbol{K}_{11}+\boldsymbol{N}_{11}-\omega_{i}^{2} \boldsymbol{M}_{11}\right) \boldsymbol{v}_{i}=0$, results in natural frequencies and the corresponding mode shapes. The vibrations of the CMFM can be obtained by superposition of these mode shapes. To gain more insight int the behaviour of the tube, the first eight mode shapes of the tube-window are depicted in Fig. 3. The first mode is a rotation of the tube-window around the $\theta_{\text {swing-axis. }}$ Later it is shown that this mode is excited when there is a massflow, due to the Coriolis effect. Therefore, the first mode is termed a Coriolis mode. The excitation of this mode, due to a mass-flow, is not at the frequency of this mode, but at the actuation frequency. The second mode is termed an in-plane mode, because it has no displacement in the direction of the sensors. The tube-window is actuated to oscillate in resonance around the $\theta_{\text {twist }}$-axis, so the third mode is termed the actuation mode. The fourth mode is also influenced by a Coriolis force and therefore termed the second Coriolis mode. The modes five and six are in-plane modes again. Mode seven is also a rotation around the $\theta_{\text {twist }}$-axis and therefore called the second actuation mode, although possible this mode is not used for actuation in our case. And finally, mode eight is again a Coriolis mode.

The reduced matrices of Eq. (4) with the first eight mode shapes $\left(\boldsymbol{V}=\left[\boldsymbol{v}_{1}, \boldsymbol{v}_{2}, \ldots, \boldsymbol{v}_{8}\right]\right)$ are derived. The reduced mass matrix is normalised to be the identity matrix:

$\boldsymbol{M}_{\text {red }}=\boldsymbol{V}^{T} \boldsymbol{M}_{11} \boldsymbol{V}=\boldsymbol{I}$

The reduced stiffness matrix is a diagonal matrix, containing the natural frequencies:

$\boldsymbol{K}_{\text {red }}=\boldsymbol{V}^{T}\left(\boldsymbol{K}_{11}+\boldsymbol{N}_{11}\right) \boldsymbol{V}=\operatorname{diag}\left(\omega_{1}^{2}, \omega_{2}^{2}, \ldots, \omega_{8}^{2}\right)$ 
The reduced velocity sensitive matrix, whereby the damping matrix $\boldsymbol{D}_{11}$ is omitted, is an skew-symmetric matrix: effect occurs at the actuation frequency $\omega_{3}$. Therefore, those modes are termed the Coriolis modes, as said before. Besides a

$$
\boldsymbol{C}_{\text {red }}=\boldsymbol{V}^{T} \boldsymbol{C}_{11} \boldsymbol{V}=\dot{\Phi}\left[\begin{array}{cccccccc}
-0.0000 & -0.0000 & -0.0279 & -0.0000 & -0.0000 & 0.0000 & 0.0047 & -0.0000 \\
0.0000 & -0.0000 & 0.0000 & -0.0000 & -0.0005 & 0.0000 & -0.0000 & 0.0000 \\
0.0279 & -0.0000 & 0.0000 & -0.0354 & -0.0000 & -0.0000 & 0.0000 & 0.0017 \\
0.0000 & 0.0000 & 0.0354 & -0.0000 & 0.0000 & -0.0000 & 0.0074 & 0.0000 \\
0.0000 & 0.0005 & 0.0000 & -0.0000 & 0.0000 & 0.0118 & 0.0000 & -0.0000 \\
-0.0000 & -0.0000 & 0.0000 & 0.0000 & -0.0118 & 0.0000 & -0.0000 & 0.0000 \\
-0.0047 & 0.0000 & -0.0000 & -0.0074 & -0.0000 & 0.0000 & 0.0000 & 0.0956 \\
0.0000 & -0.0000 & -0.0017 & -0.0000 & 0.0000 & -0.0000 & -0.0956 & 0.0000
\end{array}\right]
$$

The values of this matrix are proportional to the mass-flow $\dot{\Phi}$ trough the fluid-conveying tube. When there is no flow, this matrix is zero and there is no coupling between the modes. But when there is a flow, this matrix describes the coupling between the modes. Because this coupling is proportional to the modal velocities and the fluid velocity, this is called the Coriolis effect. The tube-window is actuated to oscillate in resonance around the $\theta_{\text {twist }}$-axis, this results mainly in a modal velocity amplitude $\dot{z}_{3}$. The third column of $\boldsymbol{C}_{\text {red }}$, expressed in Eq. (11), is examined, we see that hereby also the modes 1,4 and 8 are influenced. Whereby the mutual coupling between the symmetric and asymmetric out-ofplane modes, there is also a mutual coupling between the in-plane modes: mode 5 with mode 2 and 6 .

Predicting the Coriolis effect on tube displacements more accurate, is done by solving the quadratic eigenvalue problem:

$\left(\boldsymbol{K}_{11}+\boldsymbol{N}_{11}+j \omega_{i} \boldsymbol{C}_{11}-\omega_{i}^{2} \boldsymbol{M}_{11}\right) \boldsymbol{v}_{i}=0$

Several techniques to solve this problem are discussed by Cheesewright and Shaw [16]. They found that the eigenvalues $\omega_{i}$ are real and that the eigenvectors $\boldsymbol{v}_{i}$ are complex, because the mass, a

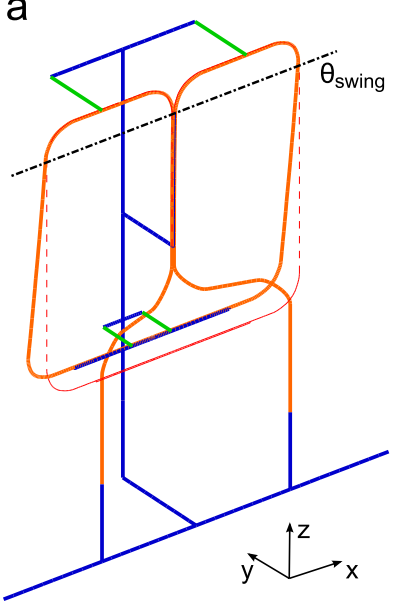

e

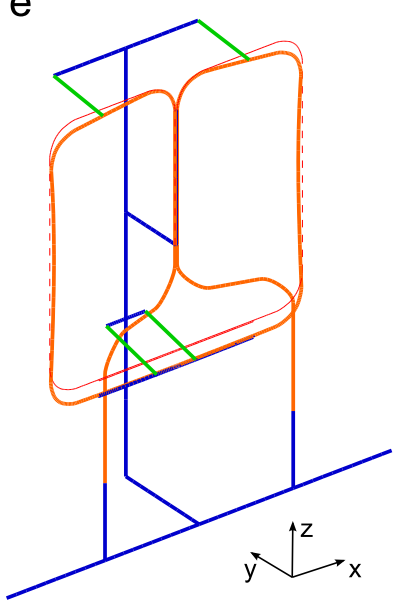

b

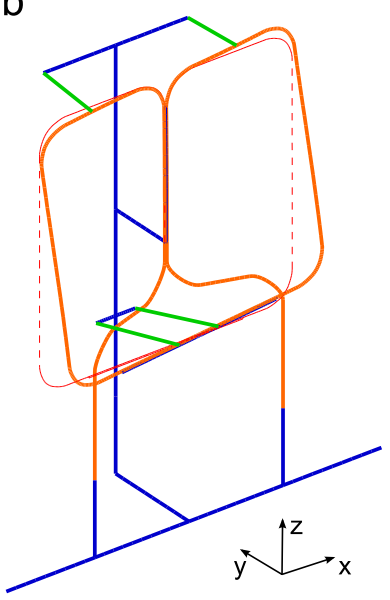

$\mathrm{f}$

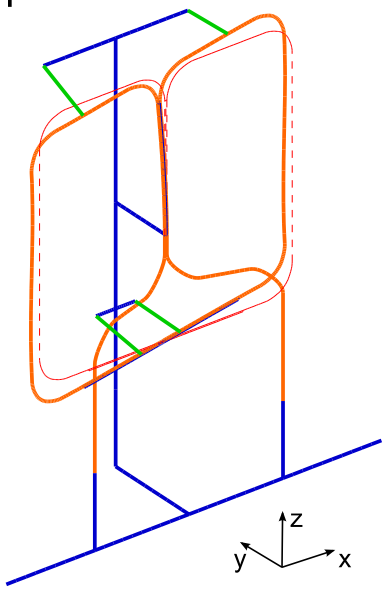

C

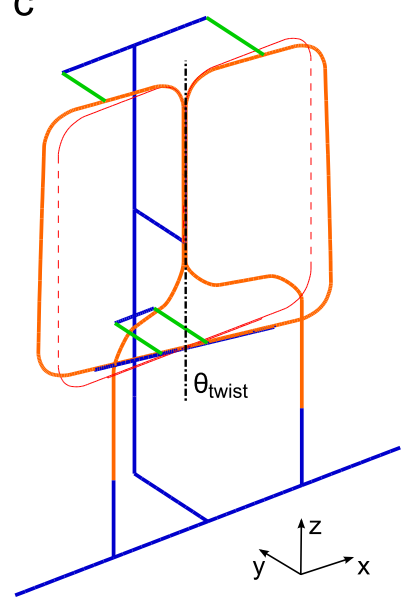

g

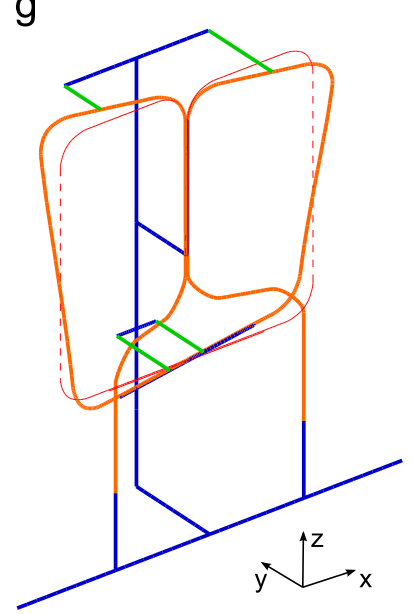

d

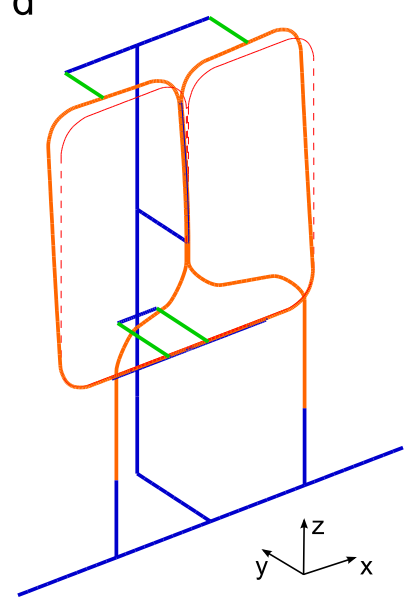

$\mathrm{h}$

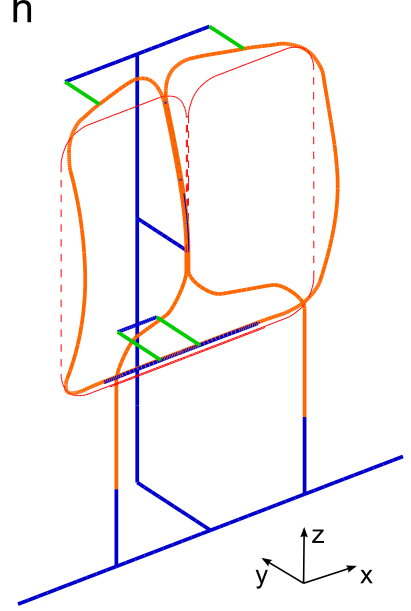

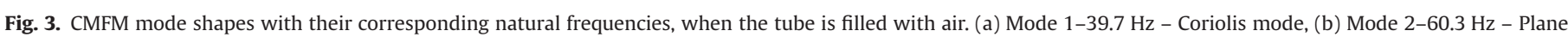

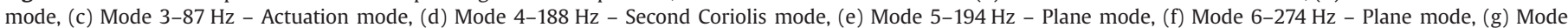
7-353 Hz - Second actuation mode, (h) Mode 8-525 Hz - Third Coriolis mode. 
a

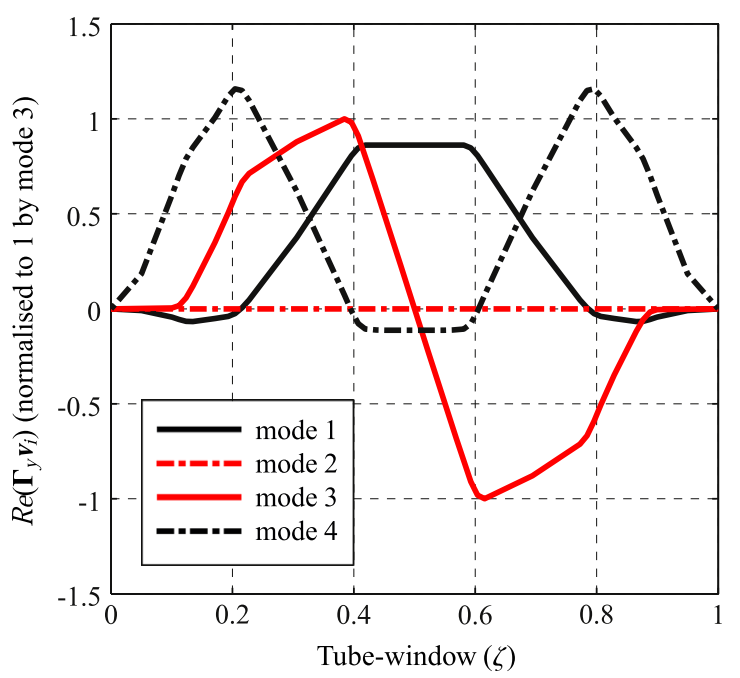

b

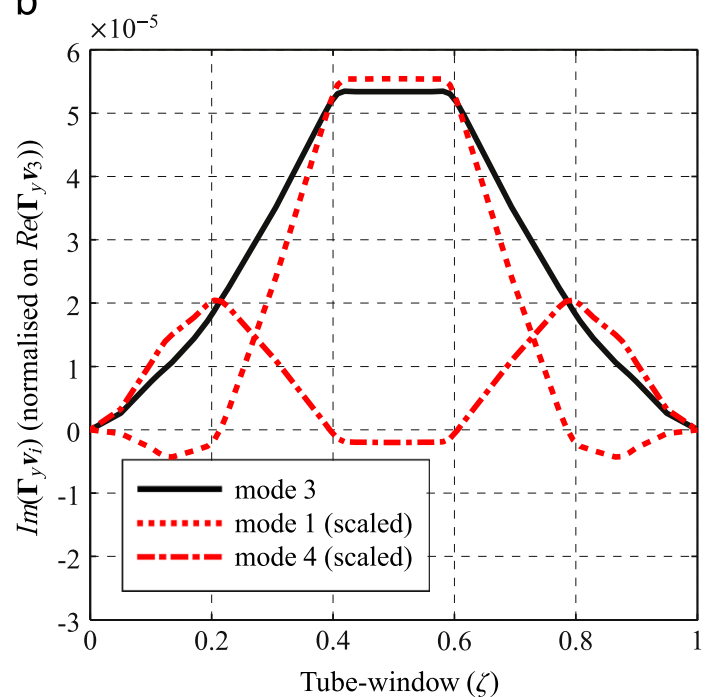

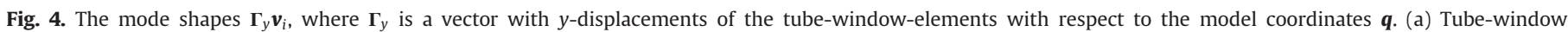

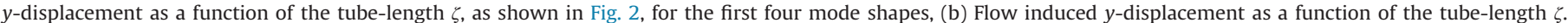
The amplitude is proportional to the mass-flow $\dot{\Phi}$.

damping and stiffness matrices are positive definite and the velocity sensitive matrix $\boldsymbol{C}_{11}$ is skew-symmetric. The real part of the mode is the conventional modes for $\dot{\Phi}=0$, while the imaginary part of the eigenvectors is the Coriolis distortion mode. The discussed techniques are unable to predict the Coriolis distortion modes accurately. We solved this issue by normalising the eigenvectors, such that $\boldsymbol{V}^{T} \boldsymbol{M}_{11} \boldsymbol{V}=\boldsymbol{I}$, resulting in a correct Coriolis distortion mode, independent of the technique used for solving the quadratic eigenvalue problem.

In Fig. 4(a) the real part of the tube-window $y$-displacement, determined from the eigenvectors, is depicted for the first four modes as a function of the tube-window center-line $\zeta$ (see Fig. 2). Where the parameter $\zeta$ follows the trajectory of the curved tubewindow, starting at the fixation point of the tube-window to the casing. The result is the same as shown in Fig. 3(a)-(d). Furthermore, in Fig. 4(b) the flow induced part of mode 3 is depicted. This is the result of solving the quadratic eigenvalue problem of Eq. (12). As suggested before, this flow induced mode can also be estimated by scaling the modes 1 and 4: $\alpha_{i} \cdot \operatorname{Re}\left(\boldsymbol{\Gamma}_{y} \boldsymbol{v}_{i}\right)$, whereby Eq. (4) is used to derive a scaling factor for those modes:

$\alpha_{i}=\frac{z_{i}}{z_{3}}=\frac{\boldsymbol{C}_{\mathrm{red}}(i, 3) \omega_{3}}{\omega_{3}^{2}-\omega_{i}^{2}} j$

where $i$ is the mode to scale. Using Eq. (11) and the natural frequencies $\omega_{i}$, we see that only the modes 1 and 4 have a significant contribution to the flow induced mode. Both scaled modes are also shown in Fig. 4(b).

The analysis above thus shows that a mass-flow only affects the out-of-phase component of the tube's motion, which can be reconstructed from scaling the other modes.

\subsection{Mass-flow measurement}

In the previous subsection the effect of a mass-flow on the mode shapes is shown. In this section it is discussed how a massflow can be measured using two displacement sensors $s_{1}$ and $s_{2}$. The tube-window is actuated to oscillate in its third eigen mode. In Fig. 4(a) we see the effect of actuation on the $y$-displacement of the tube-window. For $\zeta=0.5$, the displacement is zero, this is the rotation axis $\theta_{\text {twist }}$. In Fig. 2 we see that the sensors are placed on both sides of this rotation axis, resulting in a phase-difference between the sensor signals of $180^{\circ}$.
In Fig. 4(b) the flow induced vibration mode due to the Coriolis effect is depicted. The contribution to both sensor signals is equal in amplitude and phase, but this vibration mode occurs $90^{\circ}$ out of phase with the actuation mode, because it is the imaginary part of the mode. So, when a mass-flow is affecting the vibration mode of the tube-window, the phase-difference between the sensor signals $s_{1}$ and $s_{2}$ is not $180^{\circ}$ anymore, but is dependent on the mass-flow. The phase-difference between the two sensor signals is expressed as

$$
\begin{aligned}
\Delta \phi & =\arg \left(s_{1}\right)-\arg \left(s_{2}\right)+\pi=\arctan \left(\frac{\operatorname{Im}\left(s_{1}\right)}{\operatorname{Re}\left(s_{1}\right)}\right) \\
& -\arctan \left(\frac{\operatorname{Im}\left(s_{2}\right)}{\operatorname{Re}\left(s_{2}\right)}\right) \approx \frac{\operatorname{Im}\left(s_{1}\right)}{\operatorname{Re}\left(s_{1}\right)}-\frac{\operatorname{Im}\left(s_{2}\right)}{\operatorname{Re}\left(s_{2}\right)} \approx 2 \frac{\operatorname{Im}\left(s_{1}+s_{2}\right)}{\operatorname{Re}\left(s_{1}-s_{2}\right)}
\end{aligned}
$$

where $s_{1}$ and $s_{2}$ represent the complex displacement amplitudes, calculated solving Eq. (12). Further the first approximation $\arctan (x) \approx x)$ is valid for small radian angles only, and for the second approximation is used that the two sensors are placed on equal distance of the rotation axis $\left(\operatorname{Re}\left(s_{1}\right) \approx \operatorname{Re}\left(-s_{2}\right)\right)$. The phase difference equation is made more distinct by defining two new displacements, based on the sensor signals:

$y_{\text {act }}=\frac{1}{2}\left(s_{1}-s_{2}\right)$

$y_{\text {cor }}=\frac{1}{2}\left(s_{1}+s_{2}\right)$

where the differential-mode $s_{1}-s_{2}$ is named the actuation displacement $y_{\text {act }}$ and the common-mode $s_{1}+s_{2}$ the Coriolis displacement $y_{\text {cor }}$. This results in a new equation of the phase-difference (Eq. (14)):

$\Delta \phi \approx 2 \frac{\operatorname{Im}\left(s_{1}+s_{2}\right)}{\operatorname{Re}\left(s_{1}-s_{2}\right)}=2 \frac{\operatorname{Im}\left(y_{\text {cor }}\right)}{\operatorname{Re}\left(y_{\text {act }}\right)}$

The approximation is valid for small flows, because then the Coriolis displacement is small compared to the actuation displacement. Another advantage of this new definition is the connection with the mode shapes, presented in the previous subsection. Using Eq. (7), the actuation displacement value is written as a combination of the modal displacements:

$$
\begin{aligned}
& y_{a c t}=\frac{1}{2}\left(s_{1}-s_{2}\right)=\frac{1}{2}\left(\boldsymbol{\Gamma}_{s_{1}}-\boldsymbol{\Gamma}_{s_{2}}\right) \boldsymbol{V} \boldsymbol{z}
\end{aligned}
$$

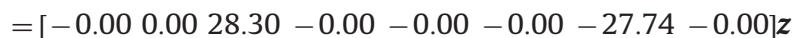


The actuation displacement is a combination of the modal amplitudes of the modes 3 and 7. The actuation modes, as presented in Fig. 3. The same holds for the Coriolis displacement, which is a combination of the modes 1,4 and 8 :

$$
\begin{aligned}
& y_{\text {cor }}=\frac{1}{2}\left(s_{1}+s_{2}\right)=\frac{1}{2}\left(\boldsymbol{\Gamma}_{s_{1}}+\boldsymbol{\Gamma}_{s_{2}}\right) \mathbf{V} \boldsymbol{z}
\end{aligned}
$$

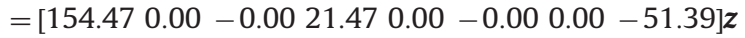

A controlled oscillation in the third mode results in excitation, proportional to the mass-flow $\dot{\Phi}$, of the modes 1,4 and 8 with the third mode frequency, see Eq. (11). The Coriolis displacement is a combination of those modal amplitudes and therefore this displacement is also proportional to the mass-flow. And, equally important, also proportional to the actuation displacement. This results in a phase difference, proportional to the mass-flow, but independent of the actuation displacement. A measurement sensitivity is defined as the phase difference per unit mass-flow:

$S=\frac{\Delta \phi}{\dot{\Phi}}[\mathrm{rad} \mathrm{s} / \mathrm{kg}]$

The mass-flow is calculated from the measured phase difference and the measurement sensitivity. The measurement sensitivity $S$ is instrument, design, fluid density and temperature dependent. In case of large flows or in the transition between laminar and turbulent the relation is non-linear and thus the sensitivity becomes also flow dependent [1]. A numerical value of the measurement sensitivity is not given for the used instrument (Fig. 1), but the phase difference $\Delta \phi$ is also a valid measure for the mass-flow as these are related.

\subsubsection{Phase demodulation}

In practice the phase of the sensor signals is measured directly, without determining the amplitudes of the sensor signals. There are different digital signal processing methods that can be applied. A method is to apply dual quadrature demodulation, the method applied to a CMFM is described by Mehendale [9]. A phase-locked loop algorithm is implemented to compute the frequency $\omega_{\text {act }}=\omega_{3}$ of the oscillating tube. The filtered frequency is used to create two waveforms: a sine and a cosine. The measured sensor signal is multiplied with both waveforms and then filtered with a low-pass filter (LPF):

$$
\begin{aligned}
s_{1} \sin \left(\omega_{a c t} t\right) & =A_{1} \sin \left(\omega_{a c t} t+\phi_{1}\right) \sin \left(\omega_{a c t} t\right) \\
& =\frac{A_{1}}{2}\left(\cos \left(\phi_{1}\right)-\cos \left(2 \omega_{a c t} t+\phi_{1}\right)\right) \stackrel{L P F}{\rightarrow}=\frac{A_{1}}{2} \cos \left(\phi_{1}\right)
\end{aligned}
$$

$$
\begin{gathered}
s_{1} \cos \left(\omega_{a c t} t\right)=A_{1} \sin \left(\omega_{a c t} t+\phi_{1}\right) \cos \left(\omega_{a c t} t\right)=\frac{A_{1}}{2}\left(\sin \left(\phi_{1}\right)\right. \\
\left.+\sin \left(2 \omega_{a c t} t+\phi_{1}\right)\right) \stackrel{L P F}{\rightarrow}=\frac{A_{1}}{2} \sin \left(\phi_{1}\right)
\end{gathered}
$$

This calculation thus results in two DC values, dependent on the phase difference $\phi_{1}$, between the sensor signal $s_{1}$ and the newly introduced waveform. Effectively the phase demodulation shifts the frequency of the sensor signals by the actuation frequency. The phase, independent of the amplitude $A_{1}$ of the sensor signals, is calculated as follows:

$\frac{\frac{A_{1}}{2} \cos \left(\phi_{1}\right)}{\frac{A_{1}}{2} \sin \left(\phi_{1}\right)}=\tan \phi_{1} \rightarrow \phi_{1}$

The same is done for the second sensor, resulting in $\phi_{2}$. This results in a phase difference between the two sensor signals:

$\Delta \phi=\phi_{1}-\phi_{2}$

The phase difference divided by the measurement sensitivity (Eq. (20)) results in an estimation of the mass-flow.
The low pass filter is the key in the trade-off between speed of the flow measurement and the measurement noise. A lower cutoff frequency reduces the amount of measurement noise, but also reduces the response time.

\subsection{Transmissibility external vibrations}

In the previous subsection we showed that the Coriolis displacement is a measure to calculate the mass-flow. In this section we show that besides a mass-flow, external vibrations result in a Coriolis displacement. The influence of external vibrations on the Coriolis displacement, expressed in the Laplace $s$-domain, is equal to

$y_{\text {cor }}(s)=\boldsymbol{T}_{y_{\text {cor }, a_{0}}}(s) \boldsymbol{a}_{0}(s)$

where $\boldsymbol{T}_{y_{\text {cor } a_{0}}}(s)$ is determined using the State Space model (Eq. (8)) and the Coriolis displacement definition (Eq. (16)). The model has in total 6 inputs, 3 translations and 3 rotations, combined in one vector $\boldsymbol{a}_{0}=\left\{a_{x}, a_{y}, a_{z}, \alpha_{R x}, \alpha_{R z}, \alpha_{R z}\right\}^{T}$. Besides the Coriolis displacement as an output, we also define the actuation displacement (Eq. (15)) as output. The MIMO system has 2 outputs and 6 inputs. This is a model with in total 12 transfer functions. The transmissibility functions of external accelerations $\boldsymbol{a}_{0}$ to the actuation and Coriolis displacements are depicted in Fig. 5.

The figure only shows three transfer functions, instead of the 12 we calculated. This is because the magnitude of the other nine is below $-300 \mathrm{~dB}$, which is approximatively zero, compared to the three remaining transfer functions.

The Coriolis displacement is influenced by a translation in $y$-direction and a rotation around the $x$-axis. Resonance frequencies are visible at the Coriolis modes (39.7, 188 and $525 \mathrm{~Hz}$ ). And the actuation displacement is influenced by a rotation around the $z$-axis. In the transfer function, resonance frequencies are visible at the actuation modes. ( 87 and $353 \mathrm{~Hz}$ ). The different modes are depicted in Fig. 3. Those three dominant directions can be explained using the couplings matrix between the modes of mechanism and the input vector:

$\boldsymbol{V}^{T} \boldsymbol{M}_{12} \boldsymbol{a}_{0}=\boldsymbol{F}_{\text {dis }} \boldsymbol{a}_{0}$
$=\left[\begin{array}{cccccc}0.00000 & -0.00703 & 0.00000 & 0.00007 & 0.00000 & -0.00000 \\ -0.00684 & -0.00000 & -0.00001 & -0.00000 & -0.00010 & 0.00018 \\ 0.00000 & 0.00000 & 0.00000 & -0.00000 & 0.00000 & -0.00013 \\ 0.00000 & 0.00598 & -0.00000 & 0.00015 & 0.00000 & -0.00000 \\ -0.00003 & 0.00000 & 0.00837 & 0.00022 & 0.00000 & 0.00000 \\ 0.00425 & 0.00000 & 0.00003 & 0.00000 & -0.00015 & -0.00011 \\ -0.00000 & 0.00000 & 0.00000 & -0.00000 & -0.00000 & -0.00002 \\ 0.00000 & 0.00151 & 0.00000 & 0.00003 & -0.00000 & -0.00000\end{array}\right] \boldsymbol{a}_{0}$

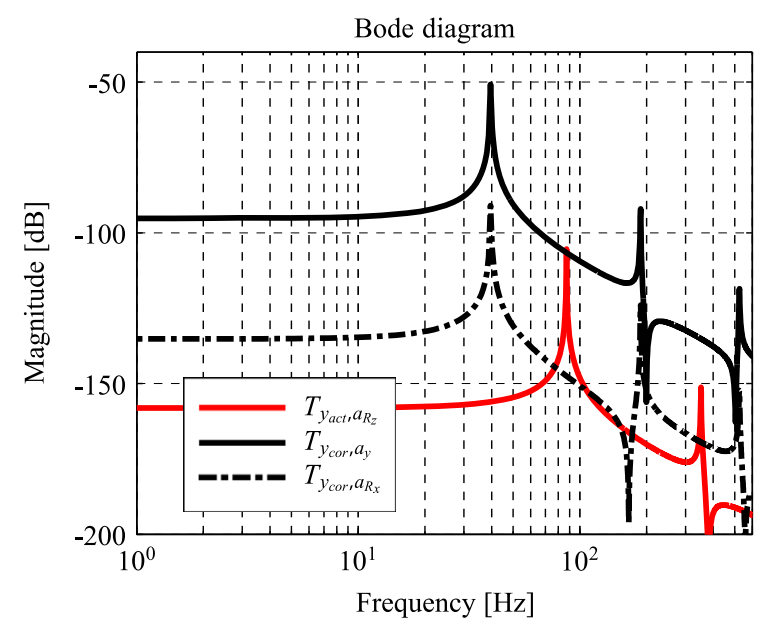

Fig. 5. Transmissibility of external vibrations to the Coriolis and actuation displacement (the rest of the transfer functions has a gain lower then $-300 \mathrm{~dB}$ ). 
The Coriolis displacement, see Eq. (19), measures only the displacements of the first, fourth and eighth mode. When we look at the rows 1,4 and 8 , we see that there are only non-zero values in the columns two and four. This indicates that the Coriolis displacement is only influenced by a translation in $y$-direction and a rotation around the $x$-axis.

The phase difference is a function of the Coriolis and actuation displacement (Eq. (17)). Those displacements is not only introduced by the actuation and due to a mass-flow, but also by external vibrations, resulting in an erroneous phase difference:

$\Delta \phi(s)=2 \frac{y_{\text {cor }}(s)}{y_{a c t}(s)} \approx \frac{2}{\left|y_{a c t}\right|} \boldsymbol{T}_{y_{c o r}, \boldsymbol{a}_{0}}(s) \boldsymbol{a}_{0}(s)$

where $\left|y_{a c t}\right|$ is the amplitude of the actuation mode. This amplitude is kept constant, using feedback control.

\subsection{Measurement error}

In Section 3.2.1, is explained how a phase difference between the sensor signals is calculated, using phase demodulation. In the frequency domain, this is similar to a bandpass filter around frequency $\omega_{3}$ and a frequency shift. First, we add the bandpass filter to Eq. (28) and obtain a transmissibility of external vibrations to the phase difference:

$\boldsymbol{T}_{\Delta \phi, \boldsymbol{a}_{0}}(s)=\frac{2}{\left|y_{\mathrm{act}}\right|} \boldsymbol{T}_{y_{\mathrm{cor}}, \boldsymbol{a}_{0}}(s) F(s)$

where $F(s)$ is a band-pass filter with a bandwidth two times the cut-off frequency of the low-pass filter, used in the phase demodulation algorithm. In Fig. 6 the dominant transmissibility including a $10 \mathrm{~Hz}$ band-pass filter around the frequency $\omega_{3}$ is depicted.

The external vibrations can be a broadband disturbance and the output is a low-frequent measurement value, due to the frequency shift. The cumulative influence is investigated by looking to the cumulative mean square response over the whole frequency range $\nu$, which is given by

$\sigma_{\Delta \phi}^{2}=\int_{0}^{\infty}\left|\boldsymbol{T}_{\Delta \phi, \boldsymbol{a}_{0}}(\nu)\right|^{2} \boldsymbol{\Phi}_{\boldsymbol{a}_{0}}(\nu) \mathrm{d} \nu$

where $\boldsymbol{\Phi}_{\boldsymbol{a}_{0}}$ is the Power Spectral Density (PSD) function of the disturbance and $\boldsymbol{T}_{\Delta \phi, \boldsymbol{a}_{0}}$ the modelled transmissibility of external vibrations to a phase difference, as partly depicted in Fig. 6 .

Due to the low-pass filter in the phase demodulation, only disturbances around the actuation frequency and the Coriolis frequency have an influence on the phase difference, see Fig. 6.

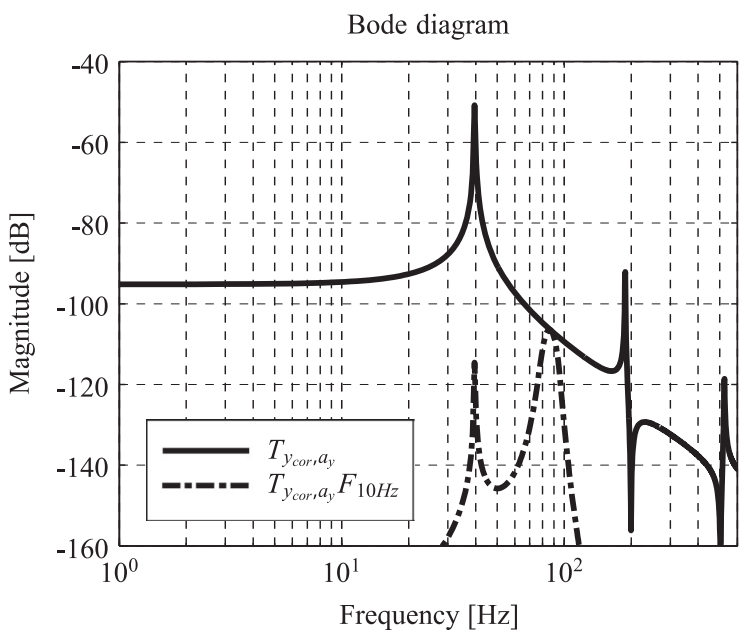

Fig. 6. Transmissibility of external vibrations in the dominant direction $a_{y}$ to a Coriolis displacement, with and without the phase demodulation including a $10 \mathrm{~Hz}$ band-pass filter.
The result is that a disturbance with a frequency close to the actuation frequency has a direct impact on a mass-flow reading. In the following section the modelled influence of external vibrations on a mass-flow measurement value is validated experimentally.

\section{Model validation}

In this section the modelled influence of external vibrations on a mass-flow measurement is validated. The first subsection explains the experimental setup. Second, the transmissibility functions of external vibrations to the actuation and Coriolis displacement are validated. Third, the influence of broadband external vibrations on the mass-flow measurement value is compared with the estimated value from the model.

\subsection{Experimental setup}

To estimate the transmissibility functions, the reference instrument is mounted on a 6-DOF vibration isolation setup (Fig. 7). The platform is suspended at a low frequency in all directions (about $22 \mathrm{~Hz}$ ), using a Stewart type platform, mounted on a rigid floor plate. The platform is actuated by six voice coil actuators. For each direction, the vibrations of the platform are measured using accelerometers. Using a rigid body model, the relation between the sensor coordinates $\boldsymbol{q}$ and Cartesian coordinates $\boldsymbol{x}=\{x, y, z$, $\left.\theta_{x}, \theta_{y}, \theta_{z}\right\}^{T}$ is derived as

$\boldsymbol{x}=\boldsymbol{R q}$

The accelerometers on the platform are colocated with the voice coil actuators. Therefore, the matrix $\boldsymbol{R}^{-1}$ is used also to apply forces in the Cartesian coordinate system. The inverse is possible because the use of 6 sensors and 6 Cartesian coordinates. More details of the experimental setup are given by Tjepkema [17]

The measurements are performed using a National Instruments NI4472 card using a $24 \mathrm{kHz}$ sample-rate with 24-bit resolution. To determine the transmissibilities, the platform is excited using the voice coil motors as shakers. These shakers provide a multi-sine signal, containing frequencies between 1 and $550 \mathrm{~Hz}$. Accelerometers (Endevco 7703A-1000) on the platform body are used to measure the input disturbance $\boldsymbol{a}_{0}$, while optic sensors inside the CMFM are used to measure the tube-window displacement.

Power Spectral Densities (PSD) of the different discrete-time signals are estimated via Welch's method. To apply the method, we use the Matlab function pwelch. In total a dataset of $60 \mathrm{~s}$ of measurement is used. To reduce the noise level, the method uses a 24k-point symmetric Hanning window. The performance is evaluated by the transmissibility function. This transfer function is estimated with the Matlab function tfestimate. Again, using a dataset of $60 \mathrm{~s}$ and a 24k-point symmetric Hanning window.

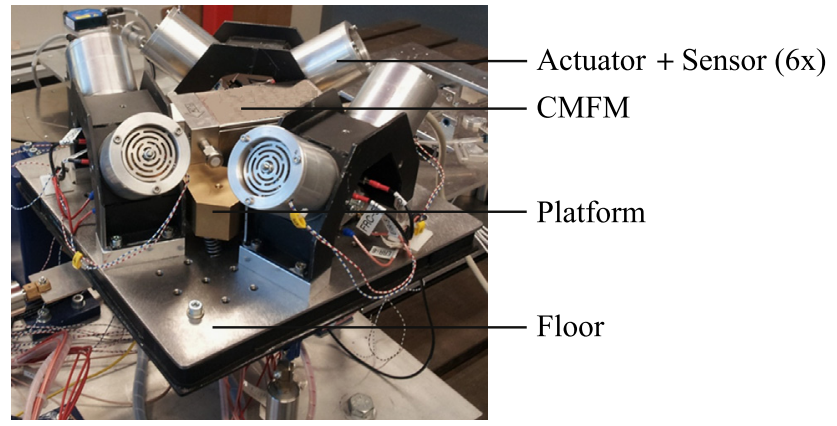

Fig. 7. Shaker setup - the CMFM (Fig. 1) is mounted on a Stewart platform. Voice coil actuators are used to apply forces on the low frequent $(22 \mathrm{~Hz})$ suspended platform and accelerometers are used to measure the platform vibrations $\boldsymbol{a}_{0}$. 


\subsection{Transmissibility}

In this subsection the transmissibilities of external vibrations to the actuation and Coriolis displacement are validated. The modelled functions are given in Section 3.3. First, the three dominant directions are estimated and second, we validate that the three functions are sufficient enough to describe the sensitivity of external vibrations.

\subsubsection{Dominant directions}

In section 3.3, we explained that are three dominant directions of the 6 external vibrations to the actuation and Coriolis displacement. In this section, we validate those three directions, using the setup described in Section 4.1. The actuators excite the platform with a random signal in one direction only and the sensors measure the displacement of the tube-window and the accelerations of the platform. Based on both datasets a transfer function is estimated. This is done for all three dominant directions.

The results of the three experiments are given in Fig. 8. The modelled results agree well with the experimental results. Clearly visible are the undamped resonance frequencies of 42 and $88 \mathrm{~Hz}$ and the gain is as expected. The estimated transmissibility $T_{y_{c o r}, \alpha_{R_{x}}}$ shows an extra peak at $22 \mathrm{~Hz}$. This is a resonance frequency of the platform. In this measurement the platform is not only rotating, but also translating in $y$-direction with frequencies mainly around the suspension frequencies. Because the transmissibility $T_{y_{c o r}, a_{y}}$ is larger than $T_{y_{\text {cor }}, \alpha_{R_{x}}}$, we cannot assume an uncoupled system and we see the effect in the estimation of $T_{y_{c o r}, \alpha_{R_{x}}}$.

\subsubsection{Broadband $3 D$ disturbance}

In the previous subsection we showed three dominant directions. Now, we need to know if those three functions are indeed the important directions. Therefore, we apply a frequency dependent force in all six directions and measure the actuation and Coriolis displacement, and the six disturbances. Now, the measured and the estimated displacement can be compared. The frequency content of a signal can be described by its Power Spectral Density (PSD), so we will compare the PSDs of both signals. The estimated PSDs are calculated as

$$
\begin{aligned}
& \hat{\boldsymbol{\Phi}}_{y_{\text {act }}}=\left|\boldsymbol{T}_{y_{\text {act }}, \boldsymbol{a}_{0}}\right|^{2} \boldsymbol{\Phi}_{\boldsymbol{a}_{0}} \approx\left|T_{y_{\mathrm{act}}, \alpha_{R_{z}}}\right|^{2} \boldsymbol{\Phi}_{\alpha_{R_{z}}} \\
& \hat{\boldsymbol{\Phi}}_{y_{\text {cor }}}=\left|\boldsymbol{T}_{y_{\mathrm{cor}}, \boldsymbol{a}_{0}}\right|^{2} \boldsymbol{\Phi}_{\boldsymbol{a}_{0}} \approx\left|T_{y_{\mathrm{cor}}, a_{y}}\right|^{2} \boldsymbol{\Phi}_{a_{y}}+\left|T_{y_{\mathrm{cor}}, \alpha_{R_{x}}}\right|^{2} \boldsymbol{\Phi}_{\alpha_{R_{x}}}
\end{aligned}
$$

The actuation displacement is, according to the model, only dependent on an $R_{z}$-disturbance, while the Coriolis displacement
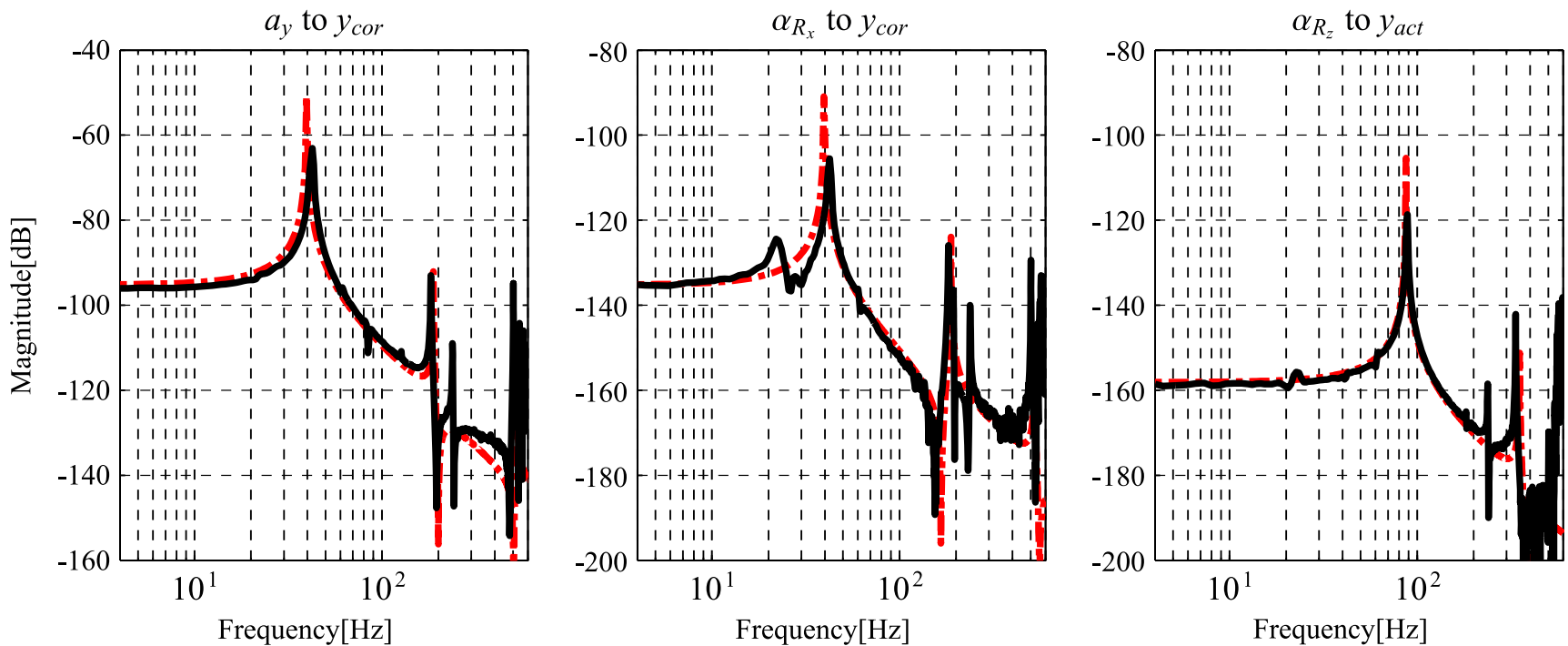

Fig. 8. Transmissibility of external vibrations to the actuation and Coriolis displacement. Experimental results as given in Fig. 5.
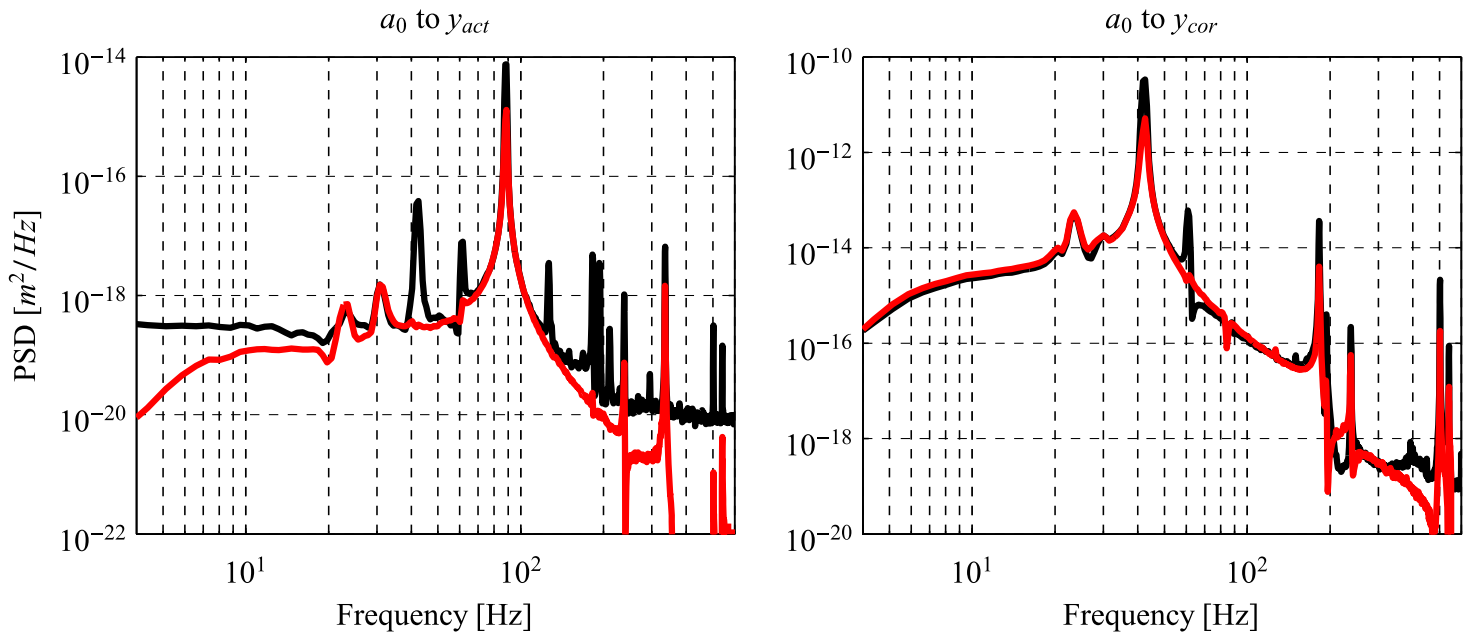

Fig. 9. Measurement (— compared to the estimation (—), based on Eqs. (31) and (32). 
is dependent on a $y$ - and $R_{x}$-disturbance. The PSDs $\boldsymbol{\Phi}_{a_{0}}$ of the applied external vibrations are shown in Fig. 10 (Experiment 4). This is an approximately broadband flat disturbance in all translation and rotational directions. Clearly visible are the resonance frequencies of the platform. The disturbance is acquired with a multi-sine disturbance between 1 and $550 \mathrm{~Hz}$. For comparison, the Vibration Criterion (VC) curves [18] are added. The VC-curves are meant as upper bounds for the peaks in the external vibration spectrum. The applied broadband disturbance is not a realistic external vibration, but is used to compare different CMFMs.

In Fig. 9 the measured PSDs of the actuation and Coriolis displacements are compared to the estimated ones (Eq. (31) and (32)), which are similar. For $\hat{\boldsymbol{\Phi}}_{y_{a c t}}$, the largest difference is that there are resonance frequencies visible at 42, 61, 88 and $183 \mathrm{~Hz}$. This clearly are modes of the system and do have an influence on the actuation displacement. In the model they do not turn up, because we assumed perfect sensors: a pure $y$-displacement and an equal sensor gain. At low and high frequencies the noise-level of the sensor is visible.

In practice the actuation displacement is of less importance, because the actuated displacement is much larger than the effect of external disturbances. For the mass-flow measurement, the Coriolis displacement is more important.

The other approximation $\hat{\boldsymbol{\Phi}}_{y_{c o r}}$ is better. The power density is equal, except a pole-zero cancellation is visible at $61 \mathrm{~Hz}$. This is an in-plane mode of the tube-window (Fig. 3(b)). This comparison confirms that there are indeed only two dominant directions, regarding the Coriolis displacement.

Another result of this experiment is that the resonance frequencies of the tube-window are estimated. The comparison, for the first

Table 1

Comparison of natural frequencies between model and experiment.

\begin{tabular}{lcll}
\hline Mode & $\begin{array}{l}\text { Model } \\
f(\mathrm{~Hz})\end{array}$ & $\begin{array}{l}\text { Experiment } \\
f(\mathrm{~Hz})\end{array}$ & Description \\
\hline 1 & 39.7 & 42.2 & Coriolis mode \\
2 & 60.3 & 61.1 & In plane mode \\
3 & 87.0 & 88.3 & Actuation mode \\
4 & 188 & 183 & Second Coriolis mode \\
5 & 194 & 193 & In plane mode \\
6 & 274 & 238 & In plane mode \\
7 & 353 & 335 & Second actuation mode \\
8 & 525 & 500 & Third Coriolis mode \\
\hline
\end{tabular}

a

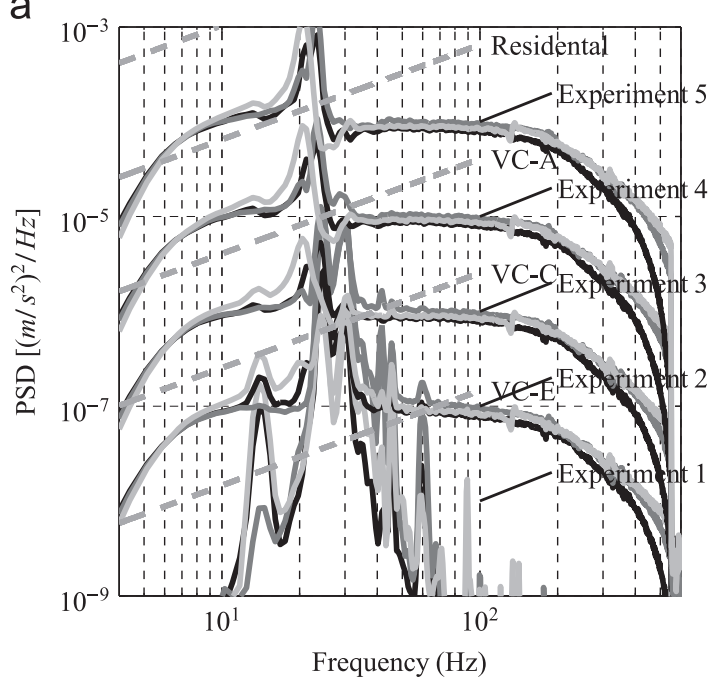

eight resonance frequencies, is given in Table 1. This comparison confirms that there is a good dynamical model available.

\subsection{Flow error}

In Section 3.4, we explained how the influence of external vibrations cumulatively add up to the RMS measurement error. In this subsection we apply disturbances in all directions with a different magnitude. Then we compare the RMS measurement value with the estimated one (Eq. (29)), to validate the influence of external vibrations. The PSDs of several experiments, with a multisine disturbance between 1 and $550 \mathrm{~Hz}$, are given in Fig. 10. Using this data, $\sigma_{\Delta \phi}$ is calculated. The results are given in Table 2. For experiment 2-5 the estimation is consistent with the measured value. In the first experiment the measured value is comparable to the noise floor of the measurement value and the estimation shows that the noise floor is larger than the effect of external vibrations.

Referring back to Fig. 6, we see that the influence is mainly due to external disturbances with frequencies around the Coriolis $(42 \mathrm{~Hz})$ and the actuation frequency of $88 \mathrm{~Hz}$. The effect around the actuation frequency can only be reduced, without affecting the mass-flow measurement, by lowering the transmissibility function $\boldsymbol{T}_{y_{c o r}, \boldsymbol{a}_{0}}$.

\section{Discussion}

In Section 4, we validated the model and saw that we can quantitatively estimate the influence of external vibrations on the

Table 2

RMS measurement error due to applied disturbance (Fig. 10), values are normalised by the first value.

\begin{tabular}{llll}
\hline Experiment & $\begin{array}{l}\text { Disturbance } \\
a_{z} \\
\text { RMS }\left(\mathrm{m} / \mathrm{s}^{2}\right)\end{array}$ & $\begin{array}{l}\text { Measurement value } \\
\text { instrument } \\
\text { RMS (rad) }\end{array}$ & $\begin{array}{l}\text { Model estimation } \\
\sigma_{\Delta \phi} \\
(\text { Eq. (29)) (rad) }\end{array}$ \\
\hline 1 & 0.0078 & 1.000 & 0.2045 \\
2 & 0.0091 & 2.037 & 1.831 \\
3 & 0.0174 & 5.680 & 5.745 \\
4 & 0.0527 & 17.63 & 18.25 \\
5 & 0.1684 & 56.20 & 56.81 \\
\hline
\end{tabular}

b

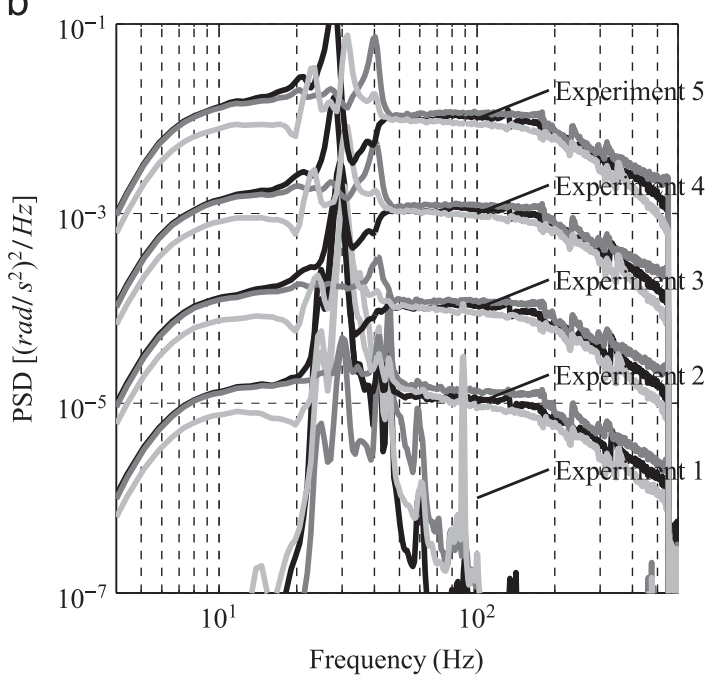

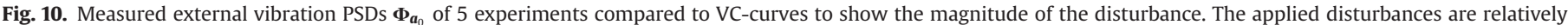
large in comparison to real floor spectra. $x(-$ ( $), y(-), z\left(-\right.$ ). (a) Translational disturbances $-a_{x}, a_{y}, a_{z}$. (b) Rotational disturbances $-\alpha_{R_{x}}, \alpha_{R_{y}}, \alpha_{R_{z}}$. 
a

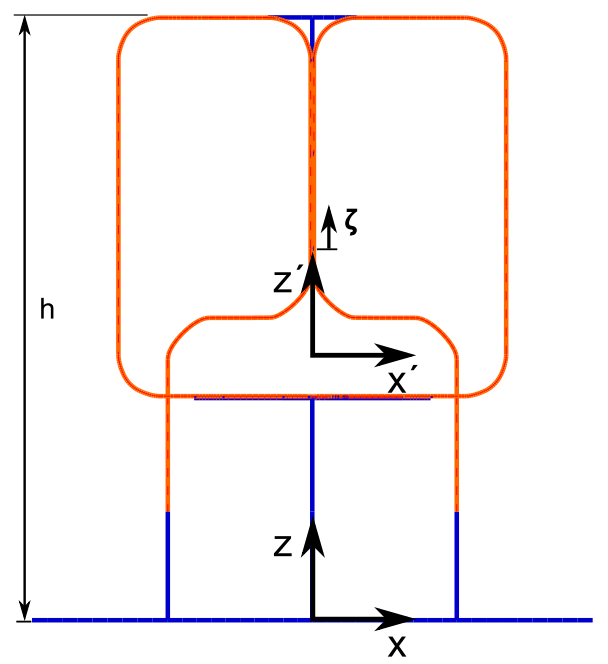

b

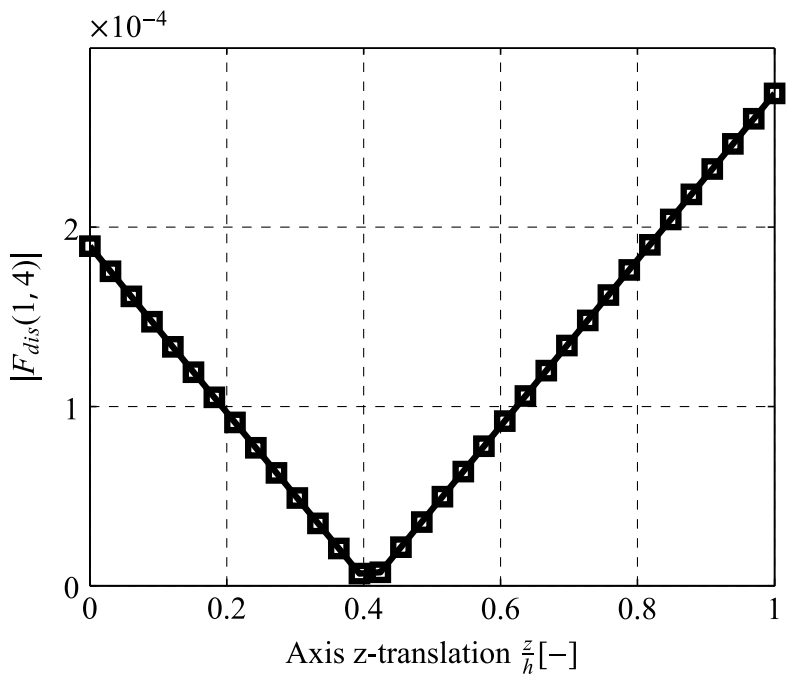

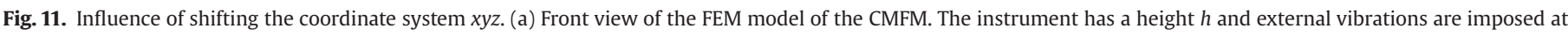

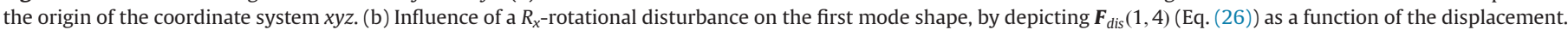

mass-flow measurement. In this section we show first that the number of relevant degrees of freedom can be reduced further and secondly how the measurement error can be reduced.

\subsection{Direction dependency}

Section 4.2 showed that the Coriolis displacement is influenced by one translational and one rotational external vibration. Especially for the rotational input, the point where the external accelerations are imposed is important. In Fig. 11(a), the front view of the CMFM FEM model (Fig. 2) is shown. At the bottom, the coordinate system $x y z$ is given. A $y$-translation and $R_{x}$-rotation result both in a Coriolis displacement, which is also an out-ofplane $y$-translation.

The flow induced displacement is mainly due to the first Coriolis mode shape, see Fig. 4(b). In Eq. (26), we saw that this mode is actuated by a $y$-translation and $R_{x}$-rotation, due to the non-zero values in the columns two and four, on the first row. Now, we can translate the coordinate system $x y z$ with a $z$-displacement, resulting in the new coordinate system $x^{\prime} y^{\prime} z^{\prime}$ (see Fig. 11(a)). Then again the matrix $\boldsymbol{F}_{\text {dis }}$ is calculated to see the effect of external vibrations on the first mode. The effect of a $R_{x}$-rotation on the first mode is shown in Fig. 11(b), by means of $\boldsymbol{F}_{\text {dis }}(1,4)$. The result shows that when the coordinate system $x y z$ is translated by $z / h \approx 0.4$, the influence of a $R_{X}$-rotation on the first mode is approximating zero. Whereby the location is dependent on the dimensional properties of the tubewindow. Note that thus the measurement of external vibration in the $y$-direction on this new point is sufficient to quantitatively estimate the RMS mass-flow error.

Therefore, the influence of external vibrations can be approximated quite well by a 1D model of one translational external vibration to the Coriolis displacement. This can be done with a mass-spring model, containing the modal mass and stiffness of the first Coriolis mode.

\subsection{Reducing influence of external vibrations}

The influence of external vibrations can be estimated, using the disturbance $\boldsymbol{\Phi}_{\boldsymbol{a}_{0}}$ and the transmissibility $\boldsymbol{T}_{\Delta \phi, \boldsymbol{a}_{0}}$ (Eq. (29)):

$\sigma_{\Delta \phi}^{2}=\int_{0}^{\infty}\left|\boldsymbol{T}_{\Delta \phi, \boldsymbol{a}_{0}}(\nu)\right|^{2} \boldsymbol{\Phi}_{\boldsymbol{a}_{0}}(\nu) \mathrm{d} \nu=\int_{0}^{\infty}\left|\frac{2}{\left|y_{a c t}\right|} \boldsymbol{T}_{y_{c o r}, \boldsymbol{a}_{0}}(\nu) F(\nu)\right|^{2} \boldsymbol{\Phi}_{\boldsymbol{a}_{0}}(\nu) \mathrm{d} \nu$
Reducing the disturbance $\boldsymbol{\Phi}_{\boldsymbol{a}_{0}}$ by implying stringent requirements on the surroundings is not possible in many applications. Therefore, the transmissibility $\boldsymbol{T}_{\Delta \phi, \boldsymbol{a}_{0}}$ should be minimal, implying a good filter algorithm and/or mechanical design of the instrument.

The transmissibility can be reduced by applying passive vibration isolation. Passive isolation consists of several stages of massspring-damper systems between the floor and the casing of a machine [19]. The parameters should be adjusted to achieve highfrequency attenuation. The reduction of the transmissibility will be subject of future research.

\section{Conclusions}

In this study a model of a CMFM is derived to understand and quantify the influence of external vibrations on the mass-flow measurement. In an experiment predefined vibrations are applied on the casing of the CMFM and the RMS measurement error is determined. The experimental results correspond well on a qualitative and quantitative level with the modelled results.

The result is an significant extension of the work of Cheesewright [8], not only the frequencies are shown where the CMFM is sensitive for external vibrations, but also a quantitative estimation of the expected mass-flow error is given, based on the modelled transmissibility function.

The agreement between model and measurements implies firstly that the influence of any external vibration spectrum on the flow error, with some limitations due to linearity of the model, can be estimated. Thereby, the suitability of a certain location for the placement of a CMFM can be determined. Secondly, the insight into the relation between vibration spectra and the flow error, the transmissibility, can be used to compare the performance of different CMFM designs and to optimise the performance by shaping their respective transfer functions.

\section{Acknowledgements}

This research was financed by the support of the Pieken in de Delta Programme of the Dutch Ministry of Economic Affairs. The authors would like to thank the industrial partner Bronkhorst High-Tech for many fruitful discussions. 


\section{References}

[1] Anklin M, Drahm W, Rieder A. Coriolis mass flowmeters: overview of the current state of the art and latest research. Flow Meas Instrum 2006;17 (6):317-23 http://dx.doi.org/10.1016/j.flowmeasinst.2006.07.004.

[2] Enz S, Thomsen J, Møller P, Davidsen L. Factors affecting Coriolis flowmeter accuracy, precision, and robustness. DCAMM special report; Technical University of Denmark (DTU); 2010. ISBN 9788790416386. URL 〈http://findit.dtu. $\mathrm{dk} / \mathrm{en} /$ catalog/112377959).

[3] Kazahaya M. A mathematical model and error analysis of coriolis mass flowmeters. Instrum Meas, IEEE Trans 2011;60(4):1163-74 http://dx.doi.org/ 10.1109/TIM.2010.2086691.

[4] Bobovnik G, Kutin J, Mole N, Štok B, Bajsi I. Numerical analysis of installation effects in coriolis flowmeters: a case study of a short straight tube full-bore design. Flow Meas Instrum 2013;34(0):142-50. http://dx.doi.org/10.1016/j. flowmeasinst.2013.10.004 URL 〈http://www.sciencedirect.com/science/arti cle/pii/S0955598613001313〉.

[5] Henry M, Tombs M, Zamora M, Zhou F. Coriolis mass flow metering for threephase flow: a case study. Flow Meas Instrum 2013;30(0):112-22. http://dx.doi org/10.1016/j.flowmeasinst.2013.01.003 URL 〈http://www.sciencedirect.com/ science/article/pii/S0955598613000095 $\rangle$.

[6] Clark C, Cheesewright R. The influence upon coriolis mass flow meters of external vibrations at selected frequencies. Flow Meas Instrum 2003;14(1-2): 33-42 http://dx.doi.org/10.1016/S0955-5986(02)00065-1.

[7] Cheesewright R, Clark C, Bisset D. The identification of external factors which influence the calibration of coriolis massflow meters. Flow Mea Instrum 2000;11(1):1-10. http://dx.doi.org/10.1016/S0955-5986(99)00023-0 URL 〈http://www.sciencedirect.com/science/article/pii/S0955598699000230〉.

[8] Cheesewright R, Belhadj A, Clark C. Effect of mechanical vibrations on coriolis mass flow meters. J Dyn Syst Meas Control 2003;125(1):103-13. http://dx.doi. org/10.1115/1.1539098
[9] Mehendale A. Coriolis mass flow rate meters for low flows [Ph.D. thesis]. University of Twente, Enschede; 2008. URL 〈http://doc.utwente.nl/60164/〉.

[10] van de Ridder L, Hakvoort W, van Dijk J, Lötters J, de Boer A. Quantitative estimation of the influence of external vibrations on the measurement error of a coriolis mass-flow meter. In: Dimitrovová Z, de Almeida RG JR. editors, 11th international conference on vibration problems (ICOVP-2013). Lisbon, Portugal; 2013. URL 〈http://doc.utwente.nl/89168/>.

[11] Jonker JB. A finite element dynamic analysis of spatial mechanisms with flexible links. Comput Methods Appl Mech Eng 1989;76(1):17-40.

[12] Jonker JB, Aarts RGKM, van Dijk J. A linearized input-output representation of flexible multibody systems for control synthesis. Multibody Syst Dyn 2009;21:99-122 http://dx.doi.org/10.1007/s11044-008-9130-6.

[13] Meijaard JP. Fluid-conveying flexible pipes modeled by large-deflection finite elements in multibody systems. J Comput Nonlinear Dyn 2013;9:011008-1-7 http://dx.doi.org/10.1115/1.4025353.

[14] Mehendale A, Lötters JC, Zwikker JM. Mass flowmeter of the coriolis type; 2006. EU Patent 1719982.

[15] Mehendale A, Lötters JC, Zwikker JM. Coriolis mass flow meter using contactless excitation and detection; 2006. EU Patent 1719983.

[16] Cheesewright R, Shaw S. Uncertainties associated with finite element modelling of coriolis mass flow meters. Flow Meas Instrum 2006;17(6):335-47 http://dx.doi.org/10.1016/j.flowmeasinst.2006.07.003.

[17] Tjepkema D. Active hard mount vibration isolation for precision equipment [Ph.D. thesis]. University of Twente, Enschede; 2012. URL 〈http://doc.utwente. $\mathrm{nl} / 82175 /\rangle$.

[18] Gordon CG. Generic vibration criteria for vibration-sensitive equipment. In: Gordon CG, editor, Society of photo-optical instrumentation engineers (SPIE) conference series, Presented at the society of photo-optical instrumentation engineers (SPIE) conference, vol. 1619; 1992. New York, p. 71-85.

[19] Rivin EI. Passive vibration isolation. ASME Press; 2003 ISBN $079810187 X$. 